\title{
Effects of Codon Usage on Gene Expression: Empirical Studies on Drosophila
}

\author{
Jeffrey R. Powell ${ }^{1} \cdot$ Kirstin Dion $^{1}$
}

Received: 16 January 2015/ Accepted: 29 March 2015/Published online: 3 April 2015

(c) The Author(s) 2015. This article is published with open access at Springerlink.com

\begin{abstract}
For most amino acids, more than one codon can be used. Many hypotheses have been put forward to account for patterns of uneven use of synonymous codons (codon usage bias) that most often have been indirectly tested primarily by analyses of patterns. Direct experimental tests of effects of synonymous codon usage are available for unicellular organisms, however empirical data addressing this problem in multicellular eukaryotes are sparse. We have developed a flexible transfecting plasmid that allows us to empirically test the effects of different codons on transcription and translation and present data from Drosophila. We could detect no significant effects of codon usage on transcription. With regard to translation, optimal codons (most used) produce higher levels of protein expression compared to non-optimal codons if the effect of difference in thermodynamic stability of secondary structure of the $5^{\prime}$ mRNA ribosome-binding site is controlled for. These results are consistent with what has been found in bacteria and thus expand the generality of these principles to multicellular eukaryotes.
\end{abstract}

Keywords Codon usage bias $\cdot$ Drosophila $\cdot$ Gene expression $\cdot$ Translation

Electronic supplementary material The online version of this article (doi:10.1007/s00239-015-9675-y) contains supplementary material, which is available to authorized users.

Jeffrey R. Powell

jeffrey.powell@yale.edu

1 Department of Ecology and Evolutionary Biology, Yale University, 21 Sachem Street, New Haven, CT 06520-8105, USA

\section{Introduction}

The genetic code is redundant: for most amino acids, more than one codon can be used to code for the same amino acid in a protein sequence. All other factors equal (such as random mutation), it is expected that over time (at equilibrium) codons for the same amino acid would be used equally frequently. But that is not the observation: codon usage is usually biased with one or two codons for an amino acid being used much more frequently than others. Which codon(s) is favored varies from taxon to taxon, that is, there is no universal pattern of codon usage bias (CUB). Why this is the case has interested molecular evolutionists for decades. Many hypotheses have been put forward to account for these observations (reviewed in Plotkin and Kudla 2011). For example, mutation bias has been given support for causing CUB in warm-blooded vertebrates that have "isochore" structured genomes (Aota and Ikemura 1986; Bulmer 1988). However, in some organisms such as Drosophila that have no isochores, mutation bias is toward A/T while codon bias is toward codons ending in $\mathrm{G} / \mathrm{C}$ (Powell and Moriyama 1997), i.e., mutation pressure is away from the most used codons. Thus other factors such as selection must be in play.

Most selection-based explanations of CUB have focused on the effects of codon usage on translation. It was hypothesized long ago that isoaccepting tRNAs (tRNAs that have the same amino acid but different anti-codons) may be in unequal abundance in the cytoplasm and this may affect efficiency of translation of different synonymous codons (Zuckerkandl and Pauling 1965; Richmond 1970). This was given support when it was documented that the most abundant tRNAs decoded most efficiently the most used codons in bacteria and yeast (Ikemura 1981, 1982) and that genes with higher protein expression had higher 
CUB (Grantham et al. 1981). In addition to speed of translation resulting in higher protein expression, Akashi (1994) provided evidence that accuracy of translation may also be involved; the most used codons result in lower levels of mis-incorporation of amino acids. The term "efficiency" of translation can be used to encompass both speed and accuracy.

In addition to affecting translation per se, codon usage may have effects on RNA structure that can affect other processes. Efficiency of splicing is one such process (Parmley and Hurst 2007). Another is the rate of mRNA ribosome binding. In bacteria, synonymous codons that change the thermodynamic stability of the secondary structure of the $5^{\prime}$ ribosome binding site of mRNA has been found to effect levels of protein expression; stronger secondary structure results in lower protein production (Kudla et al. 2009; Goodman et al. 2013).

Empirical tests of hypotheses accounting for CUB have come mostly from single-celled organisms, bacteria and yeast (Plotkin and Kudla 2011). Direct experimental test involving multi-cellular eukaryotes includes work by Carlini and Stephan (2003) and Hense et al. (2010) on the Drosophila alcohol dehydrogenase gene (Adh) and Lampson et al. (2013) on oncogene expression in human tissue culture cells.

We have developed a transfecting plasmid with cloning sites that allows us to insert a synthetic oligonucleotide of our choice and measure its effect on both transcription and translation. We tested how codon usage affects both transcription and translation in Drosophila cells including examining the role of stability of the secondary structure of the ribosome binding site. The plasmid also works in human cells and therefore potentially provides a general way to test hypotheses in a broader range of organisms.

\section{Materials and Methods}

\section{Materials and Protocol}

The experimental plasmid (Fig. 1a) was derived from the commercially available pRL-null vector (Promega, Inc.). The luciferase and SV40 region were obtained from pGL3Basic (Promega, Inc) and the Drosophila melanogaster tubulin promoter from the National Institute for Malaria Research, London. Restriction enzyme sites for the incorporation of oligos were added to either the $5^{\prime}(\mathrm{pKJ} 1)$ or $3^{\prime}$ (pKJ2) end of the firefly luciferase gene using QuikChange Lightning Site-Directed Mutagenesis (Agilent, Inc). Expression cassette II functions as our internal control and includes a renilla luciferase gene, SV40 late polyadenylation signal and actin promoter. The actin promoter was obtained from pAc5.1/V5-His (Invitrogen, Inc.). Digestions and ligations were carried out with New England
Biolabs, Inc. (NEB) products and protocols. JM109 Escherichia coli cells (Promega, Inc.) were employed in transformations.

Figure 2 shows the overall structure of the experiments. For testing single amino acids, we used the experimental oligo illustrated in Fig. 3a. Sixteen codons in sets of four for a single amino acid were tested with an arbitrary amino acid separating the sets of four. In all cases, direct sequencing confirmed the desired inserted sequence was in the correct position (Fig. 1b). In two cases, GGG for Gly and TCT for Ser, we were unable to insert the synthetic oligo possibly due to unfavorable secondary structures.

Drosophila cell lines Kc167 and S2 (obtained from the Drosophila Genomics Resource Center, Indiana University, Bloomington, Indiana, USA. http://dgrc.cgb.indiana.edu) were cultured in Schneider's $+10 \%$ FBS medium. Cells were transfected with $100 \mathrm{ng}$ plasmid diluted in enhancer buffer. Transfections were incubated at $25^{\circ} \mathrm{C}$ for $42 \mathrm{~h}$.

\section{Transcription}

RNA was isolated using the QuickExtract RNA Extraction Kit (Epicentre, Inc.) and treated with DNase I. The SensiMix Probe One-Step Kit (BioLine, Inc.) along with single tube Custom TaqMan Gene Expression Assays (Applied Biosystems, Inc.) was utilized to carry out all real time polymerase chain reactions (RT-PCR). Reactions were run in duplicate on an Applied Biosystems 7500 Fast machine, according to the following cycling conditions: one cycle at $48{ }^{\circ} \mathrm{C}$ for $30 \mathrm{~min}$, followed by 40 at $95^{\circ} \mathrm{C}$ for $1 \mathrm{~s}$, and $65^{\circ} \mathrm{C}$ for $20 \mathrm{~s}$. Means of the firefly: renilla RNA expression ratio was handled as in box in Fig. 1c.

\section{Translation}

Luciferase protein expression was evaluated with the DualGlo Luciferase Assay System (Promega, Inc.) on a Modulus Microplate Luminometer (Turner BioSystems, Inc.). Twenty microlitres of Dual-Glo Luciferase Assay Reagent was added to $20 \mu \mathrm{L}$ of each transfection and allowed to incubate at room temperature for $20 \mathrm{~min}$. Levels of firefly luciferase were then measured. Twenty microlitres of Dual-Glo Stop and Glo reagent was next added to quench firefly but activate renilla luminescence. Reactions incubated at room temperature for another $20 \mathrm{~min}$ and levels of renilla luciferase were subsequently measured. Data were handled as in Fig. 1c.

\section{Results}

Figure 1a illustrates the plasmid we have developed. It has two reporter genes to measure level of protein production; we used different promoters (tubulin and actin) to avoid 
Fig. 1 a Schematic of plasmid used in experiments. The plasmid is modified from pRLnull vector (Promega) as detailed in materials. Two cloning sites, one at each end of the protein-coding firefly luciferase gene, are shown. Distances and lengths of sequences are meant to convey general configuration and not actual distances or sizes; total length of plasmid is $10.5 \mathrm{~Kb}$. b Details of the insertion sites. c Data are handled as indicated in the box, that is, the ratio of reporter expression is normalized to the control with no inserted oligo

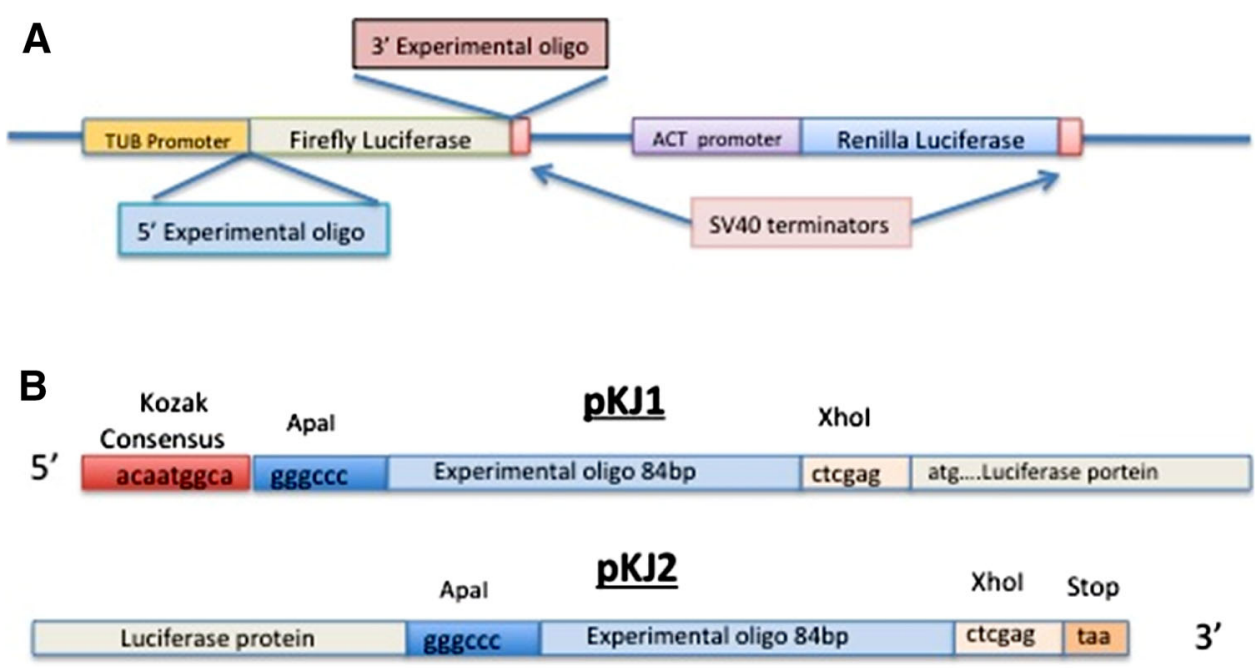

C
Fig. 2 Schematic of experimental work flow

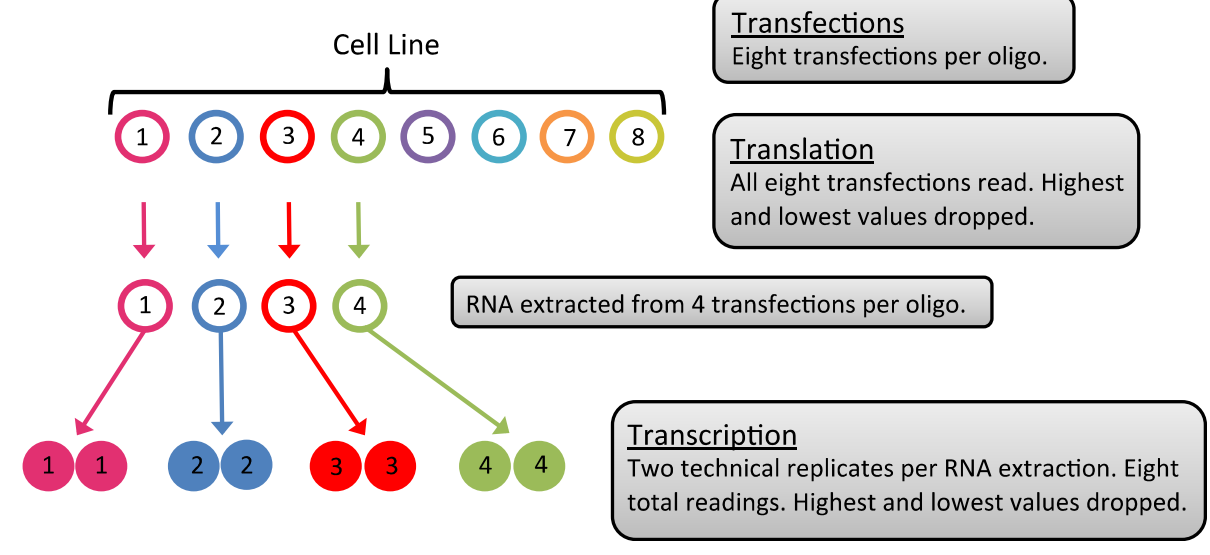

competition for transcription factors. In one reporter gene (firefly luciferase) we incorporated cloning sites at either the $5^{\prime}$ or $3^{\prime}$ end into which we inserted an experimental oligonucleotide (Fig. 1a). Drosophila tissue culture cells were transfected and level of transcription of the two reporter genes were measured by quantitative reverse PCR (qRT-PCR) and level of translation measured by reading fluorescence at two different wavelengths for the firefly and renilla luciferases. In all experiments a control was run with no inserted oligo and the data handled as indicated in the box in Fig. 1c. By basing the results on the ratio of expression of the two reporters, we control for the number of plasmids taken up in any given experiment because both reporter genes must be in the same copy number. This allows us to make comparisons between transfections. Figure 2 shows the overall structure of the experiments.

We carried out experiments following the temporal dynamics of reporter gene expression and effects of plasmid quantity (Supplementary Material Figs. S1, S2) to determine an appropriate amount of plasmid to use and time of incubation post-transfection. The data presented here used $100 \mathrm{ng}$ of plasmid analyzed $42 \mathrm{~h}$ post-transfection. 
A $a a_{1} a a_{1} a a_{1} a a_{1} S_{1} a a_{1} a a_{1} a a_{1} a a_{1} S_{2} S_{3} a a_{1} a a_{1} a a_{1} a a_{1} a a_{1} S_{4} a a_{1} a a_{1} a a_{1} a a_{1}$

B
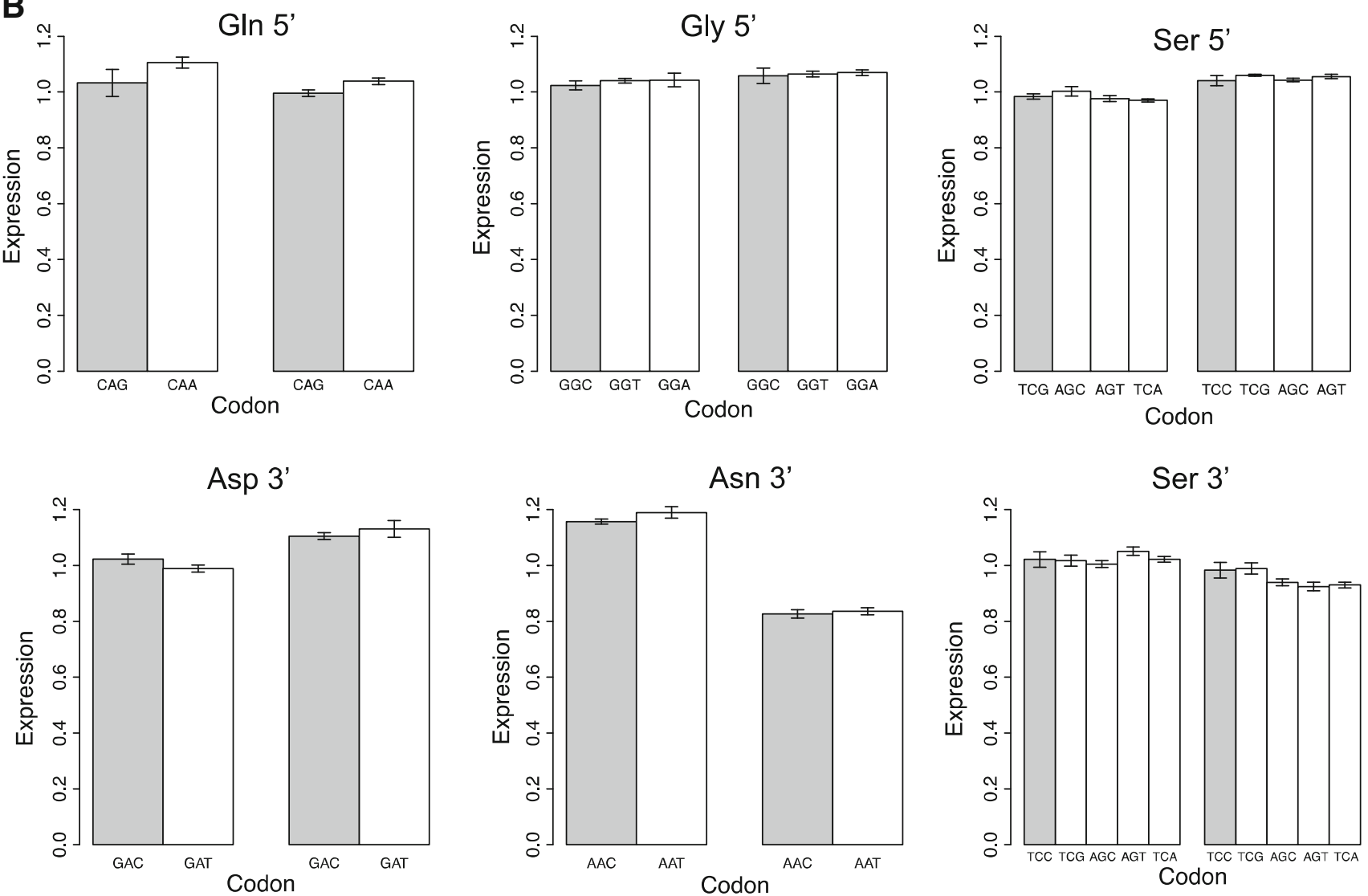

Fig. 3 a Oligo used in experiments involving single amino acids. The lower case " $\mathrm{aa}_{1}$ " represents a single amino acid with the same codon, although different experiments had different synonymous codons. $S$ is an arbitrary spacer amino acid. Sequence details in Fig. S3. b Results of qRT-PCR for mRNA expression. The codon used ("aa," above) is

Because long-term tissue culturing can induce genetic anomalies in cells, we used two D. melanogaster cell lines, Kc167 and S2 (Drosophila Genomics Resource Center, http:// dgrc.cgb.indiana.edu), to mitigate against artifacts that may occur in any single cell line.

Most of the experiments reported here were performed using experimental oligos testing the effects of codons for a single amino acid and illustrated in Fig. 3a (exact sequences in Fig. S3). Sixteen codons coding for one amino acid with the same codon were in runs of four separated by arbitrary one or two codons. Optimal and non-optimal codons were defined as in Vicario et al. (2007).

Figure 3 shows results for levels of transcription as determined by qRT-PCR. As far as detectable, codon usage had little or no effect on transcription.

Levels of protein production (translation) were affected by two factors: the codon used and the strength of the

under the bars. The optimal codon (Vicario et al. 2007) is shaded. The sets of bars on the left are for the Kc167 cell line and bars on right the S2 cell line. There are no statistically significant differences among codons except for Ser $3^{\prime}$ in the S2 cell line where TCC and TCG (left two) differ from the AGC, AGT, and TCA (right three) at $p=0.04$

secondary structure of the $5^{\prime}$ ribosome-binding site of mRNA, as previously shown for bacteria (Kudla et al. 2009). The minimum free energies (MFEs) of the predicted secondary structure of the $41 \mathrm{bp} 5^{\prime}$ ribosome-binding site were determined by software (RNA fold, http://rna.tbi.uni vie.ac.at/cgi-bin/RNAFold.cgi).

To determine robustness and repeatability of the system, we performed experiments using Ser codons in four separate experiments over several months with cells derived from independent cultures. Figure 4 shows the results. Interestingly, the two optimal codons, TCC and TCG, provide the highest level of protein expression despite having stronger secondary structure of the ribosome-binding site compared to other codons. The patterns are similar in the two cell lines. One anomaly appears to be the codon TCA which is rarely used in D. melanogaster yet is translated nearly as efficiently as the two optimal codons. The most 
Fig. 4 Replicate experiments for Ser codons. Shaded bar is the optimal codon. The cell line is $\mathrm{Kc167}$ and the insertions are in the $5^{\prime}$ end. Mean free energies ( $\mathrm{kcal} / \mathrm{mol}$ ) for the predicted secondary structure of the $41 \mathrm{bp}$ $5^{\prime}$ ribosome binding sequences are listed in order of codons in graphs. All four replicates are independent in that they were independent transfections performed with cells taken from separate cultures and in one case three months apart. Standard errors are indicated on tops of bars

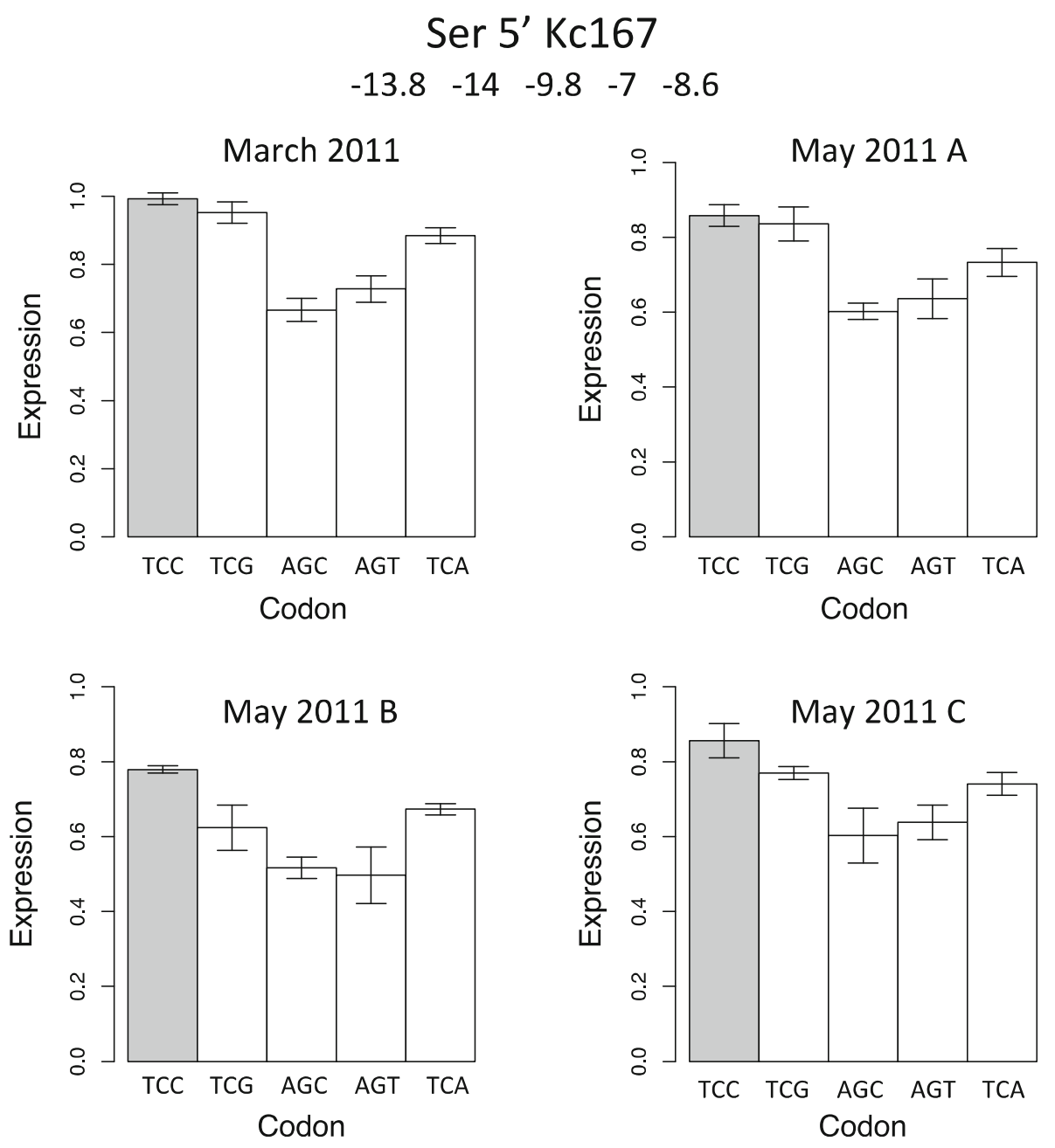

abundant tRNA $^{\text {Ser }}$ has AGA in the anticodon (Genomic tRNA database, http:/gtrnadb.ucsc.edu/Dmela/). The first A (corresponding to the wobble position) undergoes a base modification to inosine; it is known that inosine translates $\mathrm{C}$ and A about equally efficiently (Yokoyama and Nishimura 1995). Thus if this modification is greater in the tissue culture cells than in intact flies, this would explain why TCC and TCA are translated about equally efficiently.

For two amino acids, Asn and Asp, the ribosome binding sequence for the oligo with the optimal codon in the $5^{\prime}$ end had stronger or equal secondary structure than the nonoptimal codon, yet in both the $5^{\prime}$ and $3^{\prime}$ in both cell lines, the mRNA with optimal codons had higher translation (Fig. 5a). For four other amino acids, mRNAs with the oligo in the $5^{\prime}$ end with optimal codons had stronger secondary structure in the ribosome binding site than the mRNA with non-optimal codons (Fig. 5b). When in the $5^{\prime}$ end, mRNAs with the non-optimal codons with weaker secondary structure had higher or equal translation compared to the mRNAs with optimal codons. When moved to the $3^{\prime}$ end so that ribosome binding is no longer a factor, the mRNAs with optimal codons produced higher levels of protein than mRNAs with non-optimal codons.

In Figs. 4 and 5 it should be noted that in almost all cases, adding the polypeptide coded by the experimental oligo to either the $5^{\prime}$ or $3^{\prime}$ of the firefly luciferase result in a decrease in luminescence given off by the reporter, i.e., the level of luminescence is less than the control of no oligo (box in Fig. 1c). For two other amino acids we tested, Tyr and Leu, the effect of the added polypeptide on luciferase activity was too great to make reliable measurements (Supplementary Material Fig. S4).

We also tested the effect of inserting an oligo that coded for all 18 redundant amino acids; one oligo had all the optimal codons and the other all least optimal (LO) (Fig. 6). In this case the predicted MFE of the ribosome binding sites are equal $(-6.6 \mathrm{kcal} / \mathrm{mol})$. The "most optimal" (MO) was translated at higher level in both cell lines and in both the $5^{\prime}$ and $3^{\prime}$ ends compared to LO.

Statistically, we show standard errors in the figures. We also performed $t$ tests and ANOVA. Statistical significance is indicated in the figures and in captions to figures. 
A

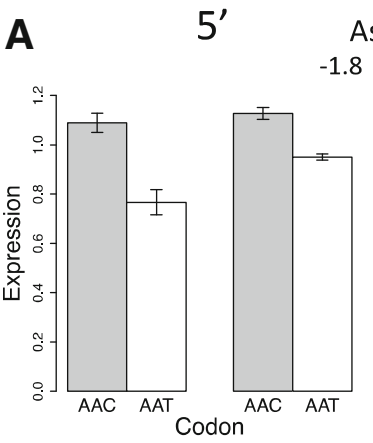

Asn

$-1.8-1.8$

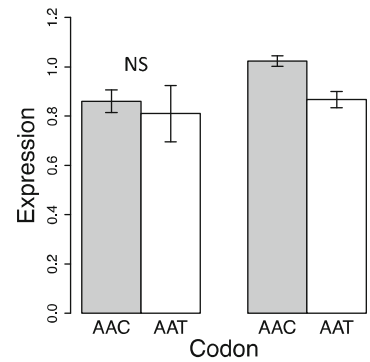

Cys

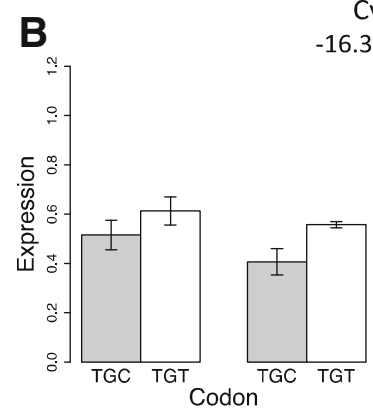

$6.3-14.6$

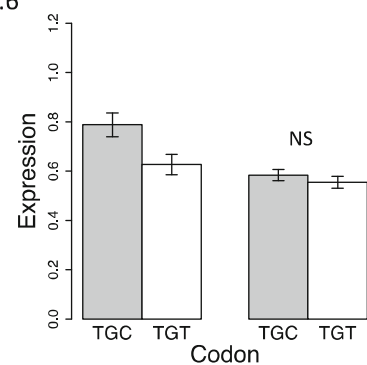

His

$-3.8-2.3$
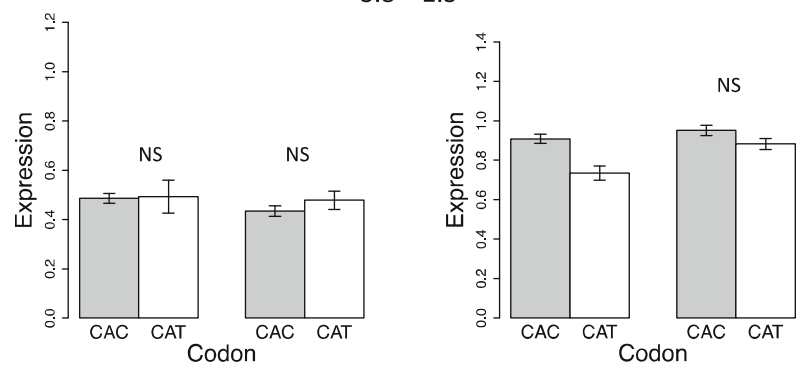

Fig. 5 a Two amino acids (Asn and Asp) where expression of the optimal codon (shaded bar) is greater in both the $5^{\prime}$ and $3^{\prime}$ ends. The minimal free energies (MFEs, $\mathrm{kcal} / \mathrm{mol}$ ) of the $41 \mathrm{bp} 5^{\prime}$ ribosome binding site is shown just below the amino acid abbreviation. b Four amino acids where the optimal codon (shaded bar) had a stronger secondary structure of the ribosome binding site (lower MFE) than

\section{Discussion}

These results are direct evidence consistent with a plethora of indirect evidence indicating optimal codons are translated more efficiently than non-optimal codons leading to higher protein product. These results are also consistent with what has been found in bacteria (Plotkin and Kudla 2011), namely that when codon usage in the $5^{\prime}$ end of messages results in differences in the strength of the secondary structure of the ribosome binding site, weaker secondary structure results in greater translation. To our knowledge, this is the first time this has been shown for a multicellular eukaryote.

The balance of the effect of secondary structure and codon usage varies among amino acids. In some cases, Ser,
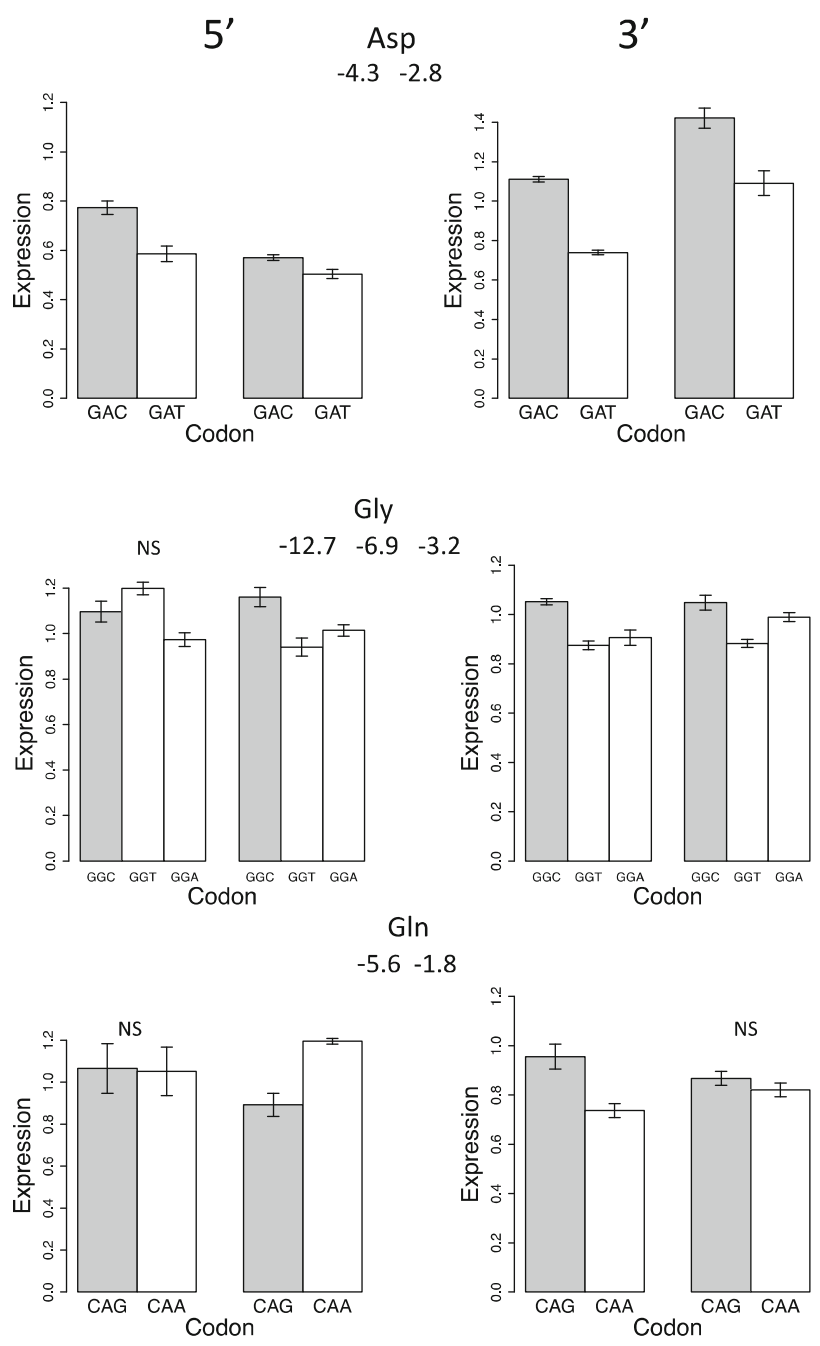

the alternative codons and where the non-optimal codon produced higher protein expression in the $5^{\prime}$ end while the optimal codon produced higher protein expression in the $3^{\prime}$ end. In all cases, the $\mathrm{Kc} 167$ cell line is on left and S2 cell line on the right and the oligo is designed as in Fig. 3a. In all cases differences between amino acids is significant at $p<0.01$ except for those with the NS indication

Asn, and Asp, even if the optimal codon in the ribosome binding site produces stronger secondary structure, protein production is greater than for the non-optimal codons (Figs. 4, 5a). In other cases (Fig. 5b) the effect of secondary structure is stronger than the effect of efficiency of translation of codons, so the effect of codon usage is dependent on position along the mRNA.

It is admittedly artificial to have four set of four identical amino acids separated by one or two codons. We designed the experiments this way to maximize the effects of codon differences for a single amino acid as we did not know how sensitive our procedure would be. It is important to emphasize that when a more realistic set of amino acids such as the oligo with one each of the redundant amino acids is tested, the results are measurable and consistent with the 
Fig. 6 Experiment with one each of the 18 amino acids coded by more than one codon. The order was randomly determined and the oligos shown in the box. In this case, the MO and LO mRNA had identical MFEs of their ribosome binding sites $-6.6 \mathrm{kcal} / \mathrm{mol}) . \mathrm{Kc} 167$ cell line on left, S2 on right. Differences are significant at $p<0.001$ for $5^{\prime}$ experiments and $p<0.01$ for $3^{\prime}$ experiments
Most Optimal (MO) TCC-GCC-GGC-TTC-AAG-ACC-GAC-GTG-CAG-CGC-AAC-GAG-CTG-TAC-ATC-TGC-CAC-CCC Amino Acid Ser-ALa-GLy-Phe-Lys-Thr-Asp-Val-GLn-Arg-Asn-GLu-Leu-Tyr-ILe-Cys-His-Pro Least Optimal (LO) AGT-GCA-GGT-TTT-AAA-ACT-GAT-GTA-CAA-AGA-AAT-GAA-CTA-TAT-ATT-TGT-CAT-CCA

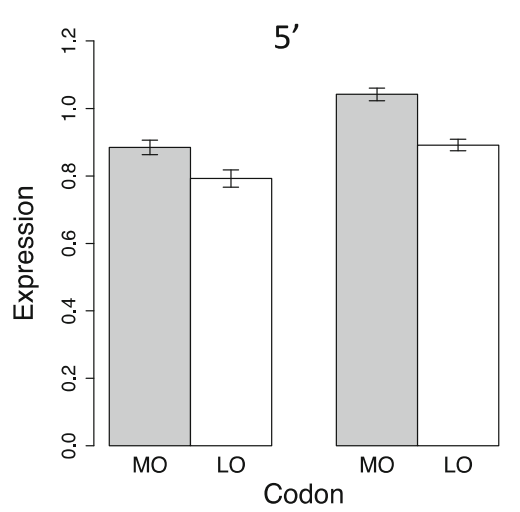

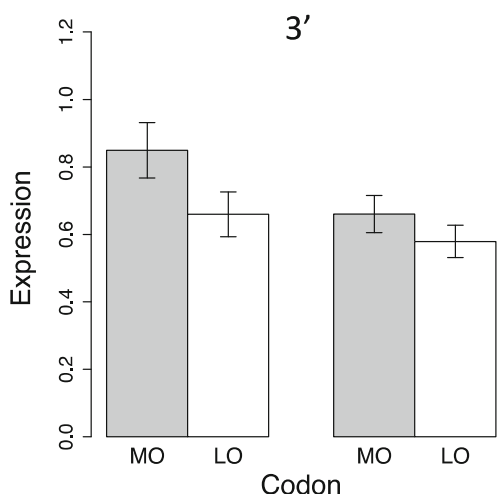

results when single amino acids are tested, i.e., optimal codons result in higher levels of protein expression (Fig. 6).

Quantitatively, our results compare favorably with the most relevant previous study, that of Carlini and Stephan (2003). They changed the codon for Leu from the optimal (codons naturally in the fly) to rarely used, presumed nonoptimal, codons. When six or ten optimal codons in the alcohol dehydrogenase $(A d h)$ gene in Drosophila were substituted with non-optimal codons, this resulted in a 19 and $24 \%$ reduction in protein expression, respectively. Six and ten codons represent 2.3 and $3.9 \%$ of the Drosophila Adh gene. In our work, 16 codons represent $2.8 \%$ of the luciferase gene. Our observed differences in expression are in the range of 5-25\%, depending on amino acid. Unfortunately, the amino acid studied by Carlini and Stephan (2003), Leu, so diminished luciferase activity as to be uninformative (Supplementary Material Fig. S4) so we could not make a more direct comparison with that previous study.

The plasmid we developed (Fig. 1a, b) is flexible in that any oligo can be inserted and the position of the experimental oligo in the expressed protein can be at either end. Having two reporters controls for efficiency of transfection, i.e., the two reporters are in the same copy number regardless of the number of plasmids taken up. Thus by treating the data as in the box in Fig. 1c, comparisons across transfections can legitimately be made. We have also tested this plasmid in a human cell line and obtained reporter expression similar to that found in Drosophila cells (Table S1), so potentially this plasmid can be used for similar studies in a broader range of organisms.

Acknowledgments We thank Jutta Roth and Saverio Vicario for advice on plasmid design and Vincent Lynch for testing the plasmid in human cells.
Open Access This article is distributed under the terms of the Creative Commons Attribution 4.0 International License (http://creativecommons.org/licenses/by/4.0/), which permits unrestricted use, distribution, and reproduction in any medium, provided you give appropriate credit to the original author(s) and the source, provide a link to the Creative Commons license, and indicate if changes were made.

\section{References}

Akashi H (1994) Synonymous codon usage in Drosophila melanogaster: natural selection and translational accuracy. Genetics 136:927-935

Aota S, Ikemura T (1986) Diversity in G+C content at the third position codons in vertebrate genes and its cause. Nucleic Acids Res 14:6345-6355

Bulmer M (1988) Codon usage and intragenic position. J Theoret Biol 133:67-71

Carlini DB, Stephan W (2003) In vivo introduction of unpreferred synonymous codons into the Drosophila Adh gene results in reduced levels of ADH protein. Genetics 163:239-243

Drosophla Genomics Resource Center, Indiana University, Bloomington. http://dgrc.cgb.indiana.edu

Goodman DB, Church GM, Kosuri S (2013) Causes and effects of $\mathrm{N}$-terminal codon bias in bacterial genes. Science 342:475-479

Grantham R, Gautier C, Gouy M, Jacobzone M, Mercier R (1981) Codon catalog usage is a genome strategy modulated for gene expressivity. Nucleic Acids Res 9:r43-r79

Hense W, Anderson N, Hutter S, Stephan W, Parsch J, Carlini D (2010) Experimentally increased codon bias in the Drosohila Adh gene leads to an increase in larval, but not adult, alcohol dehydrogenase activity. Genetics 184:547-555

Ikemura T (1981) Correlation between the abundance of Escherichia coli transfer RNAs and the occurrence of the respective codons in the protein genes: a proposal for a synonymous codon choice that is optimal for E. coli translational system. J Mol Biol 151:389-409

Ikemura $\mathrm{T}$ (1982) Correlation between the abundance of yeast transfer RNAs and the occurrence of the respective codons in protein genes: differences in synonymous codon choice patterns of yeast and Escherichia coli with reference to the abundance of isoaccepting transfer RNAs. J Mol Biol 158:573-597 
Kudla G, Murray AW, Tollervey D, Plotkin JB (2009) Coding sequence determinants of gene expression in Escherichia coli. Science 324:255-258

Lampson BL, Pershing NLK, Prinz JA, Lacsina JR, Marzluff WF, Nicchitta CV, MacAlpine DM, Counter CM (2013) Rare codons regulate KRas oncogenes. Curr Biol 23:70-75

Parmley JL, Hurst LD (2007) Exonic splicing regulatory elements skew synonymous codon usage near intron-exon boundaries in mammals. Mol Biol Evol 24:1600-1603

Plotkin JB, Kudla G (2011) Synonymous but not the same: the causes and consequences of codon bias. Nat Rev Genet 12:32-42

Powell JR, Moriyama E (1997) Evolution of codon usage in Drosophila. In Proceedings of the National Academy of Sciences USA, vol 94, pp 7784-7790
Richmond R (1970) Non-Darwinian evolution: a critique. Nature 225:1025-1028

Vicario S, Moriyama EN, Powell JR (2007) Codon usage in twelve species of Drosophila. BMC Evol Biol 7:226

Genomic tRNA Database. http://gtrnadb.ucsc.edu/Dmela/

RNA fold: http://rna.tbi.univie.ac.at/cgi-bin/RNAFold.cgi

Yokoyama S, Nishimura S (1995) Modified nucleosides and codon recognition. In: Soll D, RajBhandary D (eds) tRNA: structure, biosynthesis and function. American Society for Microbiology, Washington, pp 207-223

Zuckerkandl E, Pauling L (1965) Molecules as documents of evolutionary history. J Theoret Biol 8:357-366 